\title{
PainDroid: An Android-based Virtual Reality Application for Pain Assessment
}

\author{
Fotios Spyridonis $^{\mathrm{a}}$, Jarle Hansen ${ }^{\mathrm{a}}$, Tor-Morten Grønli ${ }^{\mathrm{b}}$ and Gheorghita Ghinea ${ }^{\mathrm{a}}$ \\ ${ }^{a}$ Department of Information Systems and Computing, Brunel University, London, \\ $U K$ \\ ${ }^{b}$ The Norwegian School of Information Technology, Oslo, Norway
}

Corresponding author: Fotios Spyridonis, Department of Information Systems and Computing, Brunel University, London, UB8 3PH, UK, Phone:

+441895265503, Email: Fotios.Spyridonis@brunel.ac.uk

\begin{abstract}
Earlier studies in the field of pain research suggest that little efficient intervention currently exists in response to the exponential increase in the prevalence of pain. In this paper, we present an Android application (PainDroid) with multimodal functionality that could be enhanced with Virtual Reality (VR) technology, which has been designed for the purpose of improving the assessment of this notoriously difficult medical concern. PainDroid has been evaluated for its usability and acceptability with a pilot group of potential users and clinicians, with initial results suggesting that it can be an effective and usable tool for improving the assessment of pain. Participant experiences indicated that the application was easy to use and the potential of the application was similarly appreciated by the clinicians involved in the evaluation. Our findings may be of considerable interest to healthcare providers, policy makers, and other parties that might be actively involved in the area of pain and VR research.
\end{abstract}

Keywords—Pain assessment, Android, Virtual Reality, 3-D Modeling, Multimodal Interfaces 


\section{Introduction}

Pain presents a significant challenge to citizens and the healthcare system of countries. Evidence from a pan-European consensus report, the Pain Proposal [1], suggests that one in five Europeans $(19 \%)$ is estimated to have some form of chronic pain, in most cases for over five years, whereas there are also cases of people who suffer for 20 years or longer.

Nonetheless, efficient intervention seems to be limited in most cases, with studies indicating a partial success of current approaches in efficiently assessing the pain experience [2 - 6]. Typical intervention practices include the visualization of pain information by using a paper-based, 2Dimensional (2-D) representation of the human body. The "pain drawing", as this representation has been named, is considered to be a valuable and useful tool in describing certain aspects of pain, such as the pain location and sensation type $[3-6,7]$. However, it can be understood that due to its 2-D format it cannot capture the 3-Dimensional (3-D) nature of the human body. As such, it can be limited in efficiently visualizing e.g. the exact location of the reported pain, something that could potentially result in the collection of inaccurate clinical information. In recognition of this, research efforts are seeking better means to address the problem.

As technology has improved and the trend has shifted to 3-D computer graphics, VR technology has nowadays become a common approach for visualization. This is the simulation of a real or imagined environment that can be experienced visually in the three dimensions of width, height, and depth, and that may additionally provide an interactive experience. Clinicians and researchers are becoming increasingly aware of the benefits that VR technology can provide in medical practice and there is now a need to introduce effective VR approaches targeted at a variety of medical conditions. To date, however, the majority of efforts in the development of VR pain applications have been focused on the management of pain in a clinical setting (i.e. hospitals), and little attention has been given to the assessment of pain and to finding ways to guide it outside clinics.

Motivated by this situation and driven by previous work by Ghinea et al. [8], in this paper we propose PainDroid: a mobile application for improved pain assessment, which has been designed to run on handheld devices (i.e. a smartphone or tablet) that run Google's Android operating system. Employing the benefits of VR technology, the PainDroid application can provide the user with a 3-D visualization of the human body that could be optionally augmented through a Head 
Mounted Display (HMD) (Figure 1), which aims to enable a user to improve their user experience and ability to report pain through a more realistic and interactive manner at any possible moment and place in time.

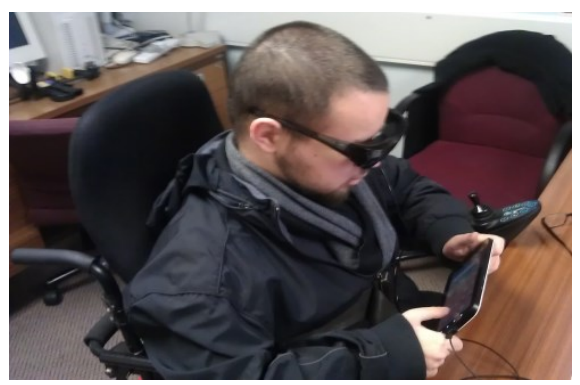

Figure 1 User evaluating the PainDroid

Accordingly, the main objective of the user study described in this paper is to identify and report disabled users' perceptions of the usability of PainDroid in pain assessment, hypothesizing that disabled peoples' mobile user experience can be improved through intuitive user interfaces that encompass novel interaction technologies, in our case, VR technology and multimodal interfaces. The rest of the paper is organized as follows. Section 2 presents background information on related research that has motivated the design of PainDroid. In section 3 , an overview of the overall design and architecture of PainDroid is provided, whereas section 4 presents the methodology employed to carry out the pilot study. Results are then described and analyzed in Section 5, whilst conclusions and future work are given in Section 6.

\section{Related Research}

\subsection{Research Motivations}

Pain is a medical concern that can be difficult to assess, as the only information that are typically used is suggestive descriptions from the patient. The pain drawing is a valid self-assessment tool that has been used since the 1940s [5] as a visual aid to enable the recording of such suggestive descriptions. A review of the pain literature performed in previous work of ours $[8-10,13]$ indicated that the pain drawing has proven to be a versatile tool for recording information as diverse as suggestive descriptions of the type and location of pain, as well as information about the existence of psychological distress and disability. In addition, our literature review revealed that pain drawings also have high completion rates and can be further used to monitor change in a patient's pain situation. 
Nevertheless, pain drawings are usually stored in a paper format, which allows no further evaluation of the data that is stored upon it, and makes searching through the data a somewhat arduous task. Moreover, their paper-based format makes them impractical to record pain variations over time, in spite of the time-dependent nature of pain [11]. To compound the issue, research work has been carried out on the use of computerized interfaces for pain assessment. Advantages of this approach over paper-based tools include the capacity to minimize errors during data transfer, the ability to record time-stamped data, ease of sharing the recorded data, and increased patient satisfaction [12].

As such, further to our previous work towards this effort $[8-10,13]$, several similar studies have been carried out that attempted to automate and improve the traditional pain assessment techniques. Such a study is the one by Jacob et al. [14], where they examined the usability of smartphone applications by children with Sickle Cell Disease for reporting their pain symptoms. From a different perspective, other studies [12, 15 - 17] proposed various electronic versions of the 2-D pain drawing to be used in the assessment of chronic pain. In all of the above studies, the results indicated that computerized interfaces have shown to be flexible and easy to use, easy to administer and complete, and have been positively endorsed by all studies' participants, supporting in this way the use of computer methods for the assessment of pain.

\subsection{Virtual Reality and Pain}

Nevertheless, the consensus of the pain literature seems to indicate that such studies rely on the 2-D representation of the pain drawing. In spite of its advantages, the 2-D pain drawing has its limitations, as it does not capture the 3-D nature of the human body. Thus, patients are unable to visually express the pain that they are experiencing, as statements of the form "I have a pain on the inside of my thigh" are not easily captured in a 2-D pain drawing, and the accuracy of the reported information can be often questioned. This has been also confirmed by a number of clinicians involved in a study by Serif and Ghinea [9], who evaluated an electronic version of the 2-D pain drawing, with their results suggesting that a 3-D human model would be more beneficial for the intended purpose than the traditional 2-D representation.

Our search in the medical literature (Medline bibliographic database: keywords "Virtual Reality" AND "Pain" (136 studies); “3D” AND "Pain” (637 studies)) revealed numerous studies which have employed 3-D technology or virtual worlds in pain research. For instance, the employment of 
VR technology as a distraction technique for burn pain care [18 - 20] is perhaps one of the most widely researched application areas. In particular, VR was found to help in reducing burn pain and distress, especially when coupled with typical analgesics. A considerable number of studies were also found dealing with the application of VR technology to the treatment of phantom limb pain [21 - 24]. Finally, VR technology has also been well-reported in a series of studies [25 - 27] to be an effective aid in reducing pain through hypnosis, as well as shown to decrease pain in persons with cancer [28 - 30]. Findings from these studies seem to suggest a high level of viability and acceptance of VR by the involved participants. However, most efforts mainly focused on the management of pain, and little (if any) work was found that attempted to employ VR technology in the assessment of pain.

As such, in the anticipation that such technology could improve the limitations of the 2-D pain drawing in pain assessment, in previous work [8, 13] the traditional 2-D pain drawing was augmented with a novel 3-D version that was designed to run both on a portable and a PC platform. Evaluations of these efforts indicated that the 3-D approach was positively received, and was thus more preferred by patients and clinicians alike, and was found particularly easy to use, as well as more precise and useful to report pain.

\subsection{Summary}

The 2-D pain drawing is a very useful and efficient tool in pain assessment. Our literature findings, however, on the use of VR technology in pain research seem to be consistent with the studies reported in a recent comprehensive review of the literature performed by Li et al. [31]. In all, these findings seem to raise three important points that need to be further addressed:

- The consensus of the literature indicates that VR technology was employed as a means to improve the management of pain, with limited attention given in studies to the application of VR for pain assessment. It seems, therefore, that there is a general absence of such technology in these efforts, and limited attempts to augment the 2-D representation of the pain drawing through VR and 3-D technology, with the exception of the authors' previous work.

- The majority of the research efforts have focused on clinic-based applications running on PC-based platforms [18-30], with only a limited number of studies paying attention to the need for mobile pain assessment. In fact, it is surprising that very few (if any) have been 
built and ported to mobile platforms. Accordingly, to the best of the authors' knowledge, there is only scarce research investigating the employment of the pain drawing for pain assessment on a mobile platform, and only a limited number of available commercial smartphone applications (e.g. the Chronic Pain Tracker, My Pain diary) [32]. Results from a study by Serif and Ghinea [9] have shown that the ability to record pain data on a mobile device could be beneficial to the participants' lifestyles, and could help see how pain varies in time. In addition, a study by Holzinger et al. [33] has further shown that patients found it easy to use a mobile application in order to record medical data.

- In most studies, there was little consideration given to the felt experience of users when they interacted with an application, and limited studies focusing on the disabled users' perspective. This is particularly important in the case of a mobile application for pain assessment of disabled people, where in order to achieve the best results in terms of user experience and ability to report pain it is crucial that the interaction with the application needs to be particularly good and realistic. In our previous work $[8,9]$ it was found that both clinicians and patients were concerned about the user experience and ability to use, due to the small font size and difficulties in interaction with handheld devices that some disabled users might face, especially those suffering from e.g. arm mobility problems.

In retrospect, considering that the application of 3-D technology on a mobile platform in the assessment of pain has been examined before, the aim of the work described in this paper is to attempt to address the above three points by proposing an alternative solution, which could further improve the previous work by utilizing two more aspects that distinguish them from previous efforts. Firstly, to tackle the issue of the small screen interface that normally a mobile device has, it is proposed that a VR component is included that would augment the small screen interface. While it could be argued that the solution to this issue could be to use a mobile device with the largest screen possible or bring the device at a comfortable self-chosen best viewing distance, this cannot always be the case with disabled users, who, in some occasions, due to their e.g. arm mobility problem might not be able to hold or move around a device that is bigger or weighs more. Consequently, it is hypothesized that using HMD equipment could improve the experience of the users by helping to achieve a satisfying field of view, while not requiring from a disabled user to relocate the device to an optimal viewing position. Several studies have previously employed a 
HMD with mobile devices as a means to reproduce a VR world for a range of purposes [34, 35]. In most cases, the HMD devices used were shown to reproduce an easy-to-navigate and use VR environment. The same feature benefit is anticipated by employing a HMD for the purpose of this study.

Secondly, improving the interfacing with the application can be an important step towards more usable VR mobile solutions. Since difficulties in interaction with a handheld device have been noted in past work, it is similarly hypothesized that enhancing the modes of interaction with the mobile solution could help the experience of users that might have mobility difficulties. Evidence from the literature seems to support the employment of additional modalities for interaction, for instance, Paggio and Jongejan [36] successfully employed gesture input to effectively interact with a 3-D avatar.

As such, in this work, we describe a user study of a mobile 3-D application for pain assessment that could be augmented through a HMD and interacted with touch and hand movement gesture input, in order to gain insights into how these improvements can affect the disabled user's experience.

\section{PainDroid Application Overview}

\subsection{Operating System}

The PainDroid application has been developed on the Android platform. The choice of this platform is grounded in the high availability of developer tools and third party libraries, and in its popularity amongst mobile device users [37]. We also needed to perform intensive 3-D model manipulation, and the 3-D libraries of Android are an integrated part of the developer environment. Moreover, the system was built with a minimum requirement of version 2.2 of Android.

As such, the developed application has been implemented using a third party 3-D library. The choice of 3-D libraries is important to be able to support advanced features of the application, such as rotating and selecting individual parts. Our choice landed on "jPCT-AE" [38], an open source, 3-D engine for Android. This tool supports all our needs, and is available free of charge for commercial, as well as personal use. Finally, one of the advantages of using this library is the possibility to run cross-device on the Android platform. Considering Android's highly fragmented composition, this demonstrates good reliability of the 3-D toolkit. 


\subsection{PainDroid Design}

The 3-D model was initially created using the Virtual Reality Modeling Language (VRML. The model consists of individually named body parts/sections that have been adapted and defined on the basis of the body regions scoring method for pain drawings described by Margolis et al. [39]. As its name suggests, in this method the human body is divided into regions and the presence of pain within a region is reported (Figure 2). The 3-D model has been, therefore, developed to allow the user to report pain on the basis of the aforementioned method.

On the screen, the user is also presented with five different pain types: numbness, stabbing, pins and needles (or tingling), burning, and stiffness (or taut), which were arbitrary color-coded. These types were chosen carefully after consultation with clinical staff, and are well-documented in the pain literature $[2,7,40]$.
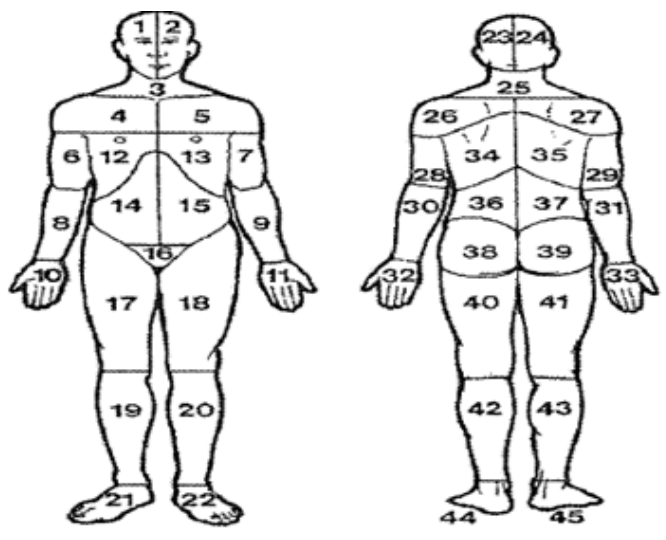

Figure 2 The Body Regions Method

User feedback from our previous research [8-10, 13] has highlighted the need for disabled users to use improved interfacing modalities for mobile pain assessment. Consequently, in PainDroid, user interaction is based both on direct touch and hand movement gesture input, in the anticipation that improving the interfacing modalities can make an important contribution to the interaction and usability of the relatively small-sized interface.

In a study by Pirhonen et al. [41], it was demonstrated that the use of specific hand gestures to control an MP3 player was indeed an effective way of interaction and more usable than the standard interface. Lumsden and Brewster [42] have also used hand gesture recognition to allow a user to interact with a Personal Digital Assistant (PDA) screen with their findings demonstrating that hand gestures have the potential to address issues concerning the usability of mobile devices. 
In similar pioneering investigations of Fitzmaurice et al. [43], user gestures and input selections were used for issuing commands in a palmtop VR condition, with the results of their research showing that applying VR concepts to palmtop computers may support effective navigation in rich and portable information spaces.

Motivated by such results, our intention with PainDroid was to concentrate on similar gestures that could be used by disabled people for interacting with the application. In order to do so, we tried to map the natural gestures a user could make when interacting with a touch screen-based smartphone to the PainDroid's interface functionality.

As a result, touch gesture input in PainDroid is implemented as follows: first, the user selects an appropriate pain type by tapping on the predefined list presented on the left of the screen, and then the location of the pain is selected, again by tapping on the desired body part of the model. The user has the option to select larger areas of the body by simply tapping on more than one such area. Each color represents a pain type and the model is colored at the selected location (Figure 3). To be able to see more details and more precisely indicate the exact pain location, pinch-to-zoom in/out, drag to move the model and flipping of the model are implemented, so as to be able to position the model at an angle and zoom level sufficient for interaction and indication of the pain location.

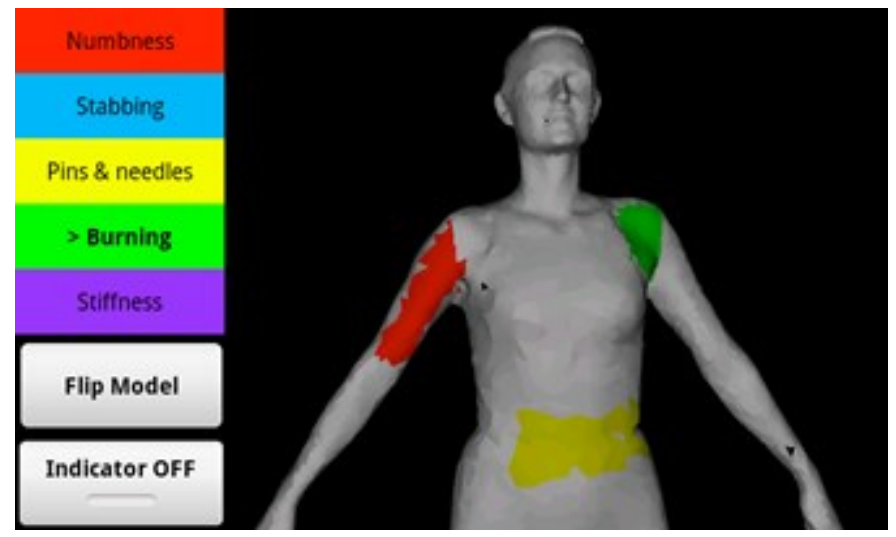

Figure 3 Interaction with the 3-D PainDroid model using touch gesture input

The user is also presented with an options menu that could be used to increase/decrease the 3-D model's rotation speed in accordance to the user's need, as well as for stopping the rotation, recentering and/or resetting the 3-D model, and finally, saving. The data that are saved consist of information about the selected body part (s) and pain type (s), as well as a timestamp that could be used to support the monitoring of the temporal evolution of pain. 


\subsection{Overall Architecture}

There are two main components responsible for the overall system architecture shown in Figure 4:

1) The views, shown in grey, and 2) the orientation listener, shown in black. We will continue by going into more detail about these two components.

Views

After presenting the initial information start screen, the main screen is shown consisting of the 3-D model, the pain types, and two buttons on the bottom left corner of the screen. This particular screen is built up using three integrated views. The first view displays the 3-D model on the screen. It has the responsibility for initializing the touch detectors responsible for all touch events, in our case consisting of: single tap to select, tap and drag to move the model, flipping the model, and pinch to zoom in/out. The options menu is also created in this view.

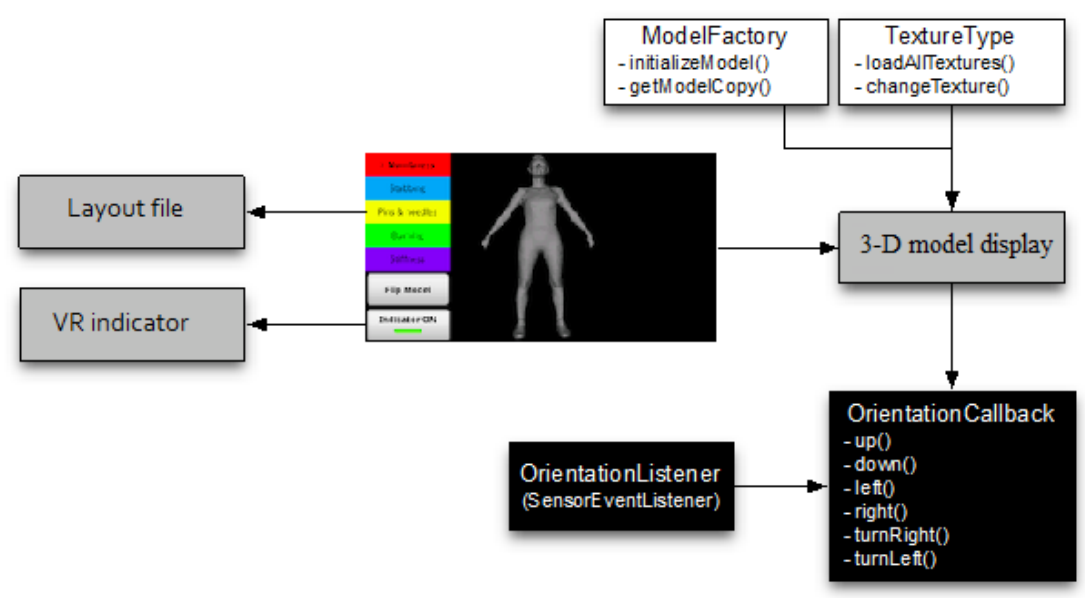

Figure 4 PainDroid overall architecture

A second view is responsible for showing the coloured buttons on the left-hand side of the screen, where the users can select the types of pain. A layout-file initializes the attributes of the buttons, like the width and height. These buttons are then responsible for changing the colours that are shown when selecting a body part on the model. The same textures used on the buttons are also implemented to colour the model when the user selects a body part.

Finally, the option to further augment the above views through VR functionality has been integrated by utilizing the benefits of an external, no head-driven VR display. In our case, this has been a set of binocular HMD glasses (Wrap 920, Vuzix), which we found to be compatible with the Android operating system. This HMD device can produce an image of 31 degree diagonal 
Field Of View (4:3 aspect ratio) through two twin high resolution (640x480, 16 million colours, $60 \mathrm{~Hz}$ progressive scan update rate) LCD displays [44]. Each participant could adjust the display by using a tilt-able section of the device.

As such, the last view is added that could be used to display a small white circle on the screen. This was included in order to clearly indicate where on the screen the touch occurred, and can be especially useful when using the HMD glasses to interact with the 3-D model in VR space. It is possible to turn on/off this feature by the toggle button called "Indicator ON/OFF".

The second main component implemented is the orientation listener, responsible for the rotation of the 3-D model. The hand movement gesture input has been employed to implement the rotation functionality of the application. In order to do so, we used the accelerometer sensor on the device. Specifically, the accelerometer was triggered when rotating the device itself, as shown in Figure 5. It only handles rotation left, right, up, and down. As such, we implemented the feature of rotating the model sideways, by tilting the device left or right, whereas tilting the device up or down would respectively rotate the model up or down.

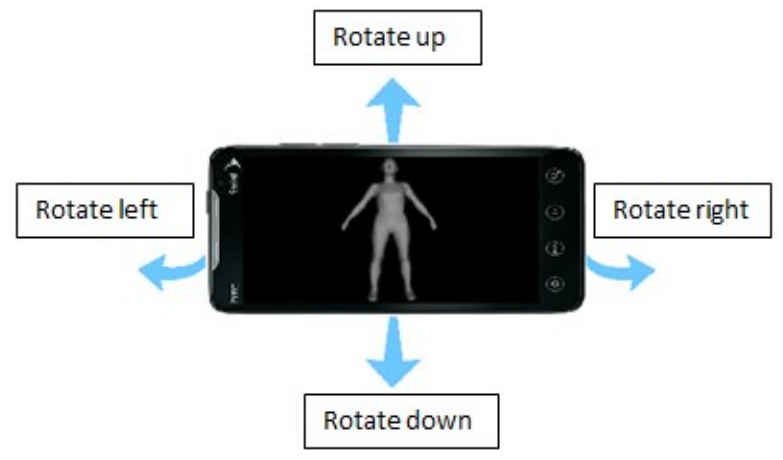

Figure 5 Interaction (rotation) with the PainDroid 3-D model using hand movement gesture input

\section{Evaluation Method and Materials}

\subsection{Participants}

The evaluation of PainDroid was performed with a convenience group of seven participants and two clinicians (a General Practitioner - GP - and a Rheumatologist - RH), who volunteered to participate in the pilot study. In particular, participants consisted of seven wheelchair users (3 females; 4 males) with some arm mobility problems, ranging in age from 21 to 65 years (mean age 41.1). All participants had previous experience of using smartphones: two were expert users, one was a very good user, and four were intermediate users (of which the two were elderly 
participants). The criteria for selection were that the participant had an age of 18 years or more, experienced some pain during the period of the study, had mild arm mobility problems, and had no previous exposure to the conventional 2-D pain drawing. The only exclusion criterion was that they should not have had any severe type of impairment, visual or otherwise, which would had prevented them from using PainDroid or experiencing its VR nature through the Vuzix HMD glasses (one user who reported that she was unable to focus on the image produced by the glasses was excluded from the study and is not included in the cohort).

While the application could be applicable to a number of pain sufferers, we had specifically targeted wheelchair users, due to the often presence of pain in these individuals, and their mobility limitations that urge for a mobile and more usable solution.

\subsection{Instrumentation and Protocol}

The pilot study was approved by the Brunel University Research Ethics Committee. Informed consent was therefore obtained by each participant prior to the evaluation. The protocol centered on the evaluation of the participants' user experience, particularly in terms of two main aspects of PainDroid: usability and acceptability. Whilst the former was examined via a questionnaire, which the wheelchair users had to fill, the latter was explored through interviews with the two clinicians involved.

The protocol followed in the user evaluation started with an initial demonstration of how to use PainDroid with and without the HMD glasses. During this initial phase, participants were also asked to freely use the application for the first time, in order to familiarize themselves with the device and the application's functionalities. Using the direct manipulation input gestures described in the previous section, each participant was then given a set of tasks to perform, but only while wearing the HMD glasses. The reason behind these decisions was that this would help us to explore the validity of our hypotheses that enhancing the modes of interaction, and using HMD equipment could both improve the experience of the disabled users by helping to achieve a satisfying ease of use and field of view, respectively, while not requiring from a user to relocate the device to an optimal viewing position for reasons explained in a previous section.

The user evaluations were performed using the HTC EVO 4G smartphone (480x800, 4.3"; 170g; $1 \mathrm{GHz} ; 512 \mathrm{MB})$. The selection of the particular device for the evaluations was based on its light weight, its satisfying screen resolution, and its fast CPU. The set of tasks that each participant was 
asked to perform consisted of the below seven tasks:

1. Starting the application

2. Rotating left/right, Rotating up/down

3. Recentering

4. Zooming in/out and dragging to all directions

5. Resetting

6. Using above to select pain type and pain location on model

7. Saving and exiting

On completion of these tasks, evaluators were then asked to complete a 15 -item questionnaire (Table 1) about the usability of PainDroid, which was adapted from the System Usability Scale (SUS) originally developed by Brooke [45]. The first 13 questions asked users to indicate on a Likert scale of 1 (Strongly Disagree) to 5 (Strongly Agree) their (dis)agreement to a series of statements regarding PainDroid, which were grouped to four different dimensions of user perception. There was a roughly equal split between positively and negatively framed questions and these were evenly distributed throughout the evaluation questionnaire. Two further questions were open-ended and asked participants to indicate what they considered to be the best aspect of PainDroid on the one hand, and to point out what they thought needed the most improvement, on the other. The seven user evaluations lasted approximately between 15-18 minutes each.

A separate evaluation was further performed with the two involved clinicians to examine the acceptability of PainDroid in medical practice. Specifically, PainDroid was demonstrated to each by walking them through the various functionalities of the application. They were then interviewed with regards to their perceptions about PainDroid's acceptance in their everyday practice in terms of how practical it could be. Each interview lasted approximately 20 minutes in duration and was recorded. These were then transcribed for analysis purposes.

\section{Evaluation Results}

We are going to structure the evaluation results according to the two stakeholder groups who participated in the study. 


\subsection{Participant Evaluation}

Reliability analysis of the responses received indicated a Cronbach alpha coefficient of 0.890, which underlies very good internal consistency. Accordingly, the participants' evaluation highlighted positive bias in respect of the application's usability and functionality.

In particular, there was relatively strong disagreement with statements targeting the amount of learning that they were required to have in order to use PainDroid, and they displayed strong agreement to the potential of other people becoming easily skillful at using it. Particularly positive responses were also received with respect to PainDroid's ease of use. The evaluation demonstrated that participants generally felt confident that it would be easy for them to use PainDroid by themselves. It is therefore reassuring that participants further indicated that they would see themselves using PainDroid whenever available.

These attitudes were also reinforced in open-ended comments targeting the best user-perceived aspects of PainDroid, for instance:

“...the ease of use, the clarity of images and versatility of the program.” (P07)

“...the relatively intuitive and clear cut.” (P02)

“...easy to use for people who have hard time writing by hand." (P05, right hand mobility

problem)

Similar encouraging responses were finally received with regards to the simplicity, clarity and helpfulness of the PainDroid's user interface. Participants were generally satisfied with the level of simplicity that the application demonstrated, they thought that it was straightforward, and thus, they liked to use the functionality provided in the user interface. The only exception to the general positive trend observed was that participants were found to be ambivalent towards the amount of functionality involved. This was reinforced in the following user comment:

“...(PainDroid) may have too much functionality in terms of manipulating the mannequin..." (P01, mobility problems to both arms)

P01, however, generally felt that the use of VR glasses to counteract the limited screen real-estate of some smartphones without the need to move them closer to an optimal viewing distance was a really good idea.

Finally, participants found that overall the different functionalities of PainDroid were well integrated, with the majority of them highlighting the good consistency of the application. 
Table 1. User Evaluation of PainDroid

\begin{tabular}{|c|c|c|c|}
\hline $\begin{array}{l}\text { Dimension of User } \\
\text { Perception }\end{array}$ & Question & Mean & SD \\
\hline \multirow[t]{6}{*}{ Ease of Use } & $\begin{array}{l}\text { Q1. I think that I would } \\
\text { like to use this } \\
\text { application frequently }\end{array}$ & 3.85 & 1.46 \\
\hline & $\begin{array}{l}\text { Q2. I felt very confident } \\
\text { using the application }\end{array}$ & 4.57 & 0.53 \\
\hline & $\begin{array}{l}\text { Q3. I thought the } \\
\text { application was easy to } \\
\text { use }\end{array}$ & 4 & 1 \\
\hline & $\begin{array}{l}\text { Q4. I think that I would } \\
\text { need the support of a } \\
\text { technical person to be } \\
\text { able to use this } \\
\text { application }\end{array}$ & 1.14 & 0.38 \\
\hline & $\begin{array}{l}\text { Q5. I found the } \\
\text { application } \\
\text { cumbersome to use } \\
\text { very }\end{array}$ & 2.57 & 1.72 \\
\hline & $\begin{array}{l}\text { Q6. I felt it difficult to } \\
\text { recover after making a } \\
\text { mistake }\end{array}$ & 1.85 & 1.21 \\
\hline \multirow[t]{2}{*}{$\begin{array}{l}\text { Consistency of } \\
\text { Application }\end{array}$} & $\begin{array}{l}\text { Q7. I found the various } \\
\text { functions in this } \\
\text { application were well } \\
\text { integrated }\end{array}$ & 4.29 & 0.49 \\
\hline & $\begin{array}{l}\text { Q8. I thought there was } \\
\text { too much inconsistency } \\
\text { in this application }\end{array}$ & 1.28 & 0.76 \\
\hline \multirow[t]{2}{*}{ Required Learning } & $\begin{array}{l}\text { Q9. I would imagine that } \\
\text { most people would learn } \\
\text { to use this application } \\
\text { very quickly }\end{array}$ & 4.14 & 1.07 \\
\hline & $\begin{array}{l}\text { Q10. I needed to learn a } \\
\text { lot of things before I } \\
\text { could get going with this } \\
\text { application }\end{array}$ & 1.14 & 0.38 \\
\hline \multirow[t]{3}{*}{$\begin{array}{l}\text { Simplicity, clarity } \\
\text { and helpfulness of UI }\end{array}$} & $\begin{array}{l}\text { Q11. I found the } \\
\text { application unnecessarily } \\
\text { complex }\end{array}$ & 2 & 1.15 \\
\hline & $\begin{array}{l}\text { Q12. I liked using the } \\
\text { interface of this } \\
\text { application }\end{array}$ & 3.85 & 1.07 \\
\hline & $\begin{array}{l}\text { Q13. The information } \\
\text { (e.g. menu) provided by } \\
\text { the application was clear } \\
\text { and helpful }\end{array}$ & 4 & 1 \\
\hline
\end{tabular}

\subsection{Clinical Evaluation}

The two clinicians involved in the PainDroid evaluation were generally quite positive towards its potential for use in a clinical setting. The following (selected) responses to the interview performed with these stakeholders reinforce this conclusion.

In our question concerning how they could use this application and how practical it could be, the GP answered that PainDroid could be:

“...used with a patient to tell me where they're hurting...in the consultation room..." 
“...very useful in the consultation room for mapping pain... and could be used to compare over time to see the improvement..."

Accordingly, the RH's responses to the same question were that Paindroid could be:

“...advantageous if you can keep in memory previous recordings and patients don't know that - then you can keep on seeing how pain has changed...that would be quite neat!"

“...useful, since the idea of sending pain information to the doctor, directly from the app, results in patients getting more out of the doctor's appointment due to saving time."

Both clinicians were also asked their opinion on how patients could potentially use the application, and whether it could have any influence in better assessing pain. It is heartening to note that both clinicians, as well as the participants, appreciated the potential of PainDroid as a pain assessment tool, highlighting two key areas, of medication intake and patient-clinician dialogue:

“...patients can use it to assess whether their medication was working and to assess whether things were getting better or worse with the treatment that was offered, like physiotherapy..." (GP)

“...really great idea, opens up the possibility of rich dialogue between patients and clinicians.” (P01)

Moreover, in order to examine their perceptions of PainDroid from a technological point of view, both clinicians were asked to indicate what they liked and/or disliked about PainDroid, and if they had any suggestions for improvement. It was particularly encouraging to find out that the RH really appreciated the multimodal interface of PainDroid, since, he believed, patients with neurological illness and/or with limited hand mobility could potentially use the improved sensor-based interface to better interact with the application. However, they both mentioned that it might need some fine-tuning in order to make it more applicable to a wider range of disabled users.

Finally, in our last question on whether PainDroid could be used in everyday medical practice, there was a mutual agreement on the application's potential in everyday pain assessment; however, as both the GP and RH mentioned, the prototypical nature of PainDroid means that acceptance by the community could also potentially encounter hurdles: 
“...I'm still coming to grips with the fact that it is a very different way of looking at things..." (RH)

“...it's too early, a lot of doctors are not used to adopting tools like this, but eventually when they get it on board they will start to use it and can become very widespread very quickly.” (GP)

\section{Conclusions and Future Work}

Pain is a prevalent, but sometimes, underemphasized medical concern. Whilst there is an abundance of sophisticated, but expensive clinical tools and apparatuses to investigate the underlying physiological causes, there is a scarcity of tools which address self-expressed dimensions of pain. In this paper, we have addressed the latter concern and have described PainDroid, a prototypical Android-based multimodal and VR application for pain assessment.

The present pilot study's results support the potential of using computerized interfaces for the assessment of pain and are in agreement with findings presented in previous similar studies [12, 14 - 17] about their ease of use and acceptance by the involved participants. In particular, our pilot evaluation with both clinicians and a group of wheelchair-using participants with mild arm mobility problems has highlighted a generally positive attitude towards the usability of PainDroid's novel functionality, as well as the potential of the application to open up new avenues of patient-clinician interaction through automating a patient's pain diary.

Our evaluation has furthermore produced important results with respect to the future development of the application. Specifically, responses from our evaluation with the clinicians indicated that PainDroid could be used to "compare pain over time" (GP) and "assess whether things were getting better or worse" (GP). In doing so, clinicians suggested that the application should be able to further provide a means to report pain parameters (e.g. severity and/or duration) other than just the location and type of pain, or to include additional pain types. These would be equally important when used by clinicians for the aforementioned purposes. The above functionalities constitute part of our future efforts for further development of the application.

As such, we acknowledge that the application is in a prototypical stage, and has only been evaluated with a relatively small sample of participants and clinicians that is not sufficient to currently generalize our results. Our findings, nonetheless, reveal encouraging signs of both stakeholders' acceptance and satisfaction with the developed proof-of-concept. Further 
development, larger scale evaluations, and further assessment of clinical efficacy all form part of our future efforts.

\section{Acknowledgements}

The authors would like to acknowledge Cyberware, Inc. for providing a 3-D model, which we adapted and then manipulated and extended to meet this study's needs. We also acknowledge EDB ErgoGroup for providing employee time to evaluate our application.

\section{References}

[1] M. Baker et al. Improving the current and future management of chronic pain: A European Consensus Report [online] Available: http://www.mijnpijn.nl/pdf/PainProposalEuropeanReport.pdf. Accessed on: 27/07/2012

[2] Lee, SJ (2001) Pain measurement: Understanding existing tools and their application in the emergency department. Emerg Med 13: 279-287.

[3] Mannion, AF, Balague, F, Pellise, F, Cedraschi, C (2007) Pain measurement in patients with low back pain. Nat Clin Pract Rheumatol 3: 610-618.

[4] Haefeli, M, Elfering, A (2006) Pain assessment. Eur Spine J 15: 17-24.

[5] Ohnmeiss, DD (2000) Repeatability of Pain Drawings in a Low Back Pain Population. Spine 25: 980-988.

[6] Jamison, RN, Fanciullo, GJ, Baird, JC (2004) Usefulness of Pain Drawings in Identifying Real or Imagined Pain: Accuracy of Pain Professionals, Non-professionals, and a Decision Model. J Pain 5: 476-482.

[7] Masferrer, R, Prendergast, V, Hagell, P (2003) Colored pain drawings: preliminary observations in neurosurgical practice. Eur J Pain. 7: 213-217.

[8] Ghinea, G, Spyridonis, F, Serif, T, Frank, AO (2008) 3-D pain drawings: mobile data collection using a PDA. IEEE Trans Inf Technol Biomed 12: 27-33.

[9] Serif, T, Ghinea, G (2005) Recording of Time-Varying Back-Pain Data: A Wireless Solution. IEEE Trans Inf Technol Biomed 9: 447-458.

[10] Serif, T, Ghinea, G, Frank, AO (2005) Visualizing pain data for wheelchair users: a ubiquitous approach. J Mob Multimed 1:161-177. 
[11] Gibson, J, Frank, AO. Pain experienced by Electric Powered Indoor/Outdoor Chairs (EPIOC) users: A pilot exploration using pain drawings. in Proc. 2nd Meeting Eur. Fed. Phys. Rehab. Med., Vienna, Austria, May 2004, p. 114.

[12]Lalloo, C, Henry, JL (2011) Evaluation of the Iconic Pain Assessment Tool by heterogeneous group of people in pain. Pain Res Manag 16:13-18.

[13] Spyridonis, F, Gawronski, J, Ghinea, G, Frank, AO (2012) An interactive 3D application for pain management: results from a pilot study in spinal cord injury rehabilitation. Comput Methods Programs Biomed. In press.

[14] Jacob, E et al (2012) Usability Testing of a Smartphone for Accessing a Web-based e-Diary for Self-monitoring of Pain and Symptoms in Sickle Cell Disease. J Pediatr Hematol Oncol 34: 326-335.

[15] Jamison, RN, Fanciullo, GJ, Baird, JC (2004) Computerized Dynamic Assessment of Pain: Comparison of Chronic Pain Patients and Healthy Controls. Pain Med 5: 168-177.

[16] Marceau, LD, Link, C, Jamison, RN, Carolan, S (2007) Electronic Diaries as a Tool to Improve Pain Management: Is There Any Evidence? Pain Med 8: S101-S109.

[17] Von Baeyer, CL, Lin, V, Seidman, LC, CI Tsao, J, Zeltzer, LK (2011) Pain charts (body charts or manikins) in assessment of the location of pediatric pain. Pain Manag 1: 61-68.

[18] Maani, CV et al (2011) Combining ketamine and virtual reality pain control during severe burn wound care: one military and one civilian patient. Pain Med 12: 673-678.

[19] Maani, CV et al (2011) Virtual reality pain control during burn wound debridement of combat-related burn injuries using robot-like arm mounted VR goggles. J Trauma 71: S125130.

[20] Hoffman, HG et al (2011) Virtual reality as an adjunctive non-pharmacologic analgesic for acute burn pain during medical procedures. Ann Behav Med 41:183-191.

[21] Gaggioli, A, Amoresano, A, Gruppioni, E, Verni, G, Riva, G (2010) A myoelectric-controlled virtual hand for the assessment and treatment of phantom limb pain in trans-radial upper extremity amputees: a research protocol. Stud Health Technol Inform 154: 220-222.

[22] Murray, CD et al (2007) The treatment of phantom limb pain using immersive virtual reality: three case studies. Disabil Rehabil 29: 1465-1469. 
[23] Cole, J, Crowle, S, Austwick, G, Slater, DH (2009) Exploratory findings with virtual reality for phantom limb pain; from stump motion to agency and analgesia. Disabil Rehabil 31: 846854.

[24] Lamont, K, Chin, M, Kogan, M (2011) Mirror box therapy: seeing is believing. Explore 7: $369-372$.

[25] Patterson, DR, Tininenko, JR, Schmidt, AR, Sharar, SR (2004) Virtual reality hypnosis: a case report. Int J Clin Exp Hypn 52: 27-38.

[26] Patterson, DR, Jensen, MP, Wiechman, SA, Sharar, SR (2010) Virtual reality hypnosis for pain associated with recovery from physical trauma. Int J Clin Exp Hypn 58: 288-300.

[27] Patterson, DR, Wiechman, SA, Jensen, MP, Sharar, SR (2006) Hypnosis delivered through immersive virtual reality for burn pain: A clinical case series. Int J Clin Exp Hypn 54:130142.

[28] Sander Wint, S, Eshelman, D, Steele, J, Guzzetta, CE (2002) Effects of distraction using virtual reality glasses during lumbar punctures in adolescents with cancer. Oncol Nurs Forum 29: E8-E15.

[29] Windich-Biermeier, A, Sjoberg, I, Dale, JC, Eshelman, D, Guzzetta, CE (2007) Effects of distraction on pain, fear and distress during venous port access and venipuncture in children and adolescents with cancer. J Pediatr Oncol Nurs 24: 8-19.

[30]Kwekkeboom, KL, Cherwin, CH, Lee, JW, Wanta, B (2010) Mind-body treatments for the pain-fatigue-sleep disturbance symptom cluster in persons with cancer. J Pain Symptom Manag 39: 126-138

[31]Li, A, Montano, Z, Chen, VJ, Gold, JI (2011) Virtual reality and pain management: current trends and future directions. Pain Manag 1: 147-157.

[32]Rosser, BA, Eccleston, C (2011) Smartphone applications for pain management. J Telemed Telecare 17: 308-312.

[33] Holzinger, A, Kosec, P, Schwantzer, G, Debevc, M, Frühwirth, J, Hofmann-Wellenhof, R. (2011). Design and Development of a Mobile Computer Application to Reengineer Workflows in the Hospital and the Methodology to Evaluate its Effectiveness. J Biomed Inform 44 (6): 563-570. 
[34] Basogain, X, Izkara, JL, Borro. D. Educational Mobile Environment with Augmented Reality Technology. In Proc Int Technol Educ Dev Conf, 7-9 March 2007, Valencia.

[35] Basu, A, Raij, A, Johnsen, K. Ubiquitous Collaborative Activity Virtual Environments. ACM Conf Comput Support Cooperat Work. 11-15 Febraury 2012, Seattle, Washington.

[36] Paggio, P, Jongejan, B. Multimodal Communication in the Virtual Farm of the Staging Project. In Proc Int Workshop Inf Present Natural Multimod Dialogue, Verona, 2001, pp. 41 45.

[37] Holzinger, A, Treitler, P, Slany, W. (2012). Making Apps Useable on Multiple Different Mobile Platforms: On Interoperability for Business Application Development on Smart phones. In: G. Quirchmayr et al. (Eds.): CD-ARES 2012, Springer Lecture Notes in Computer Science LNCS 7465, IIFIP International Federation for Information Processing, 176 - 189

[38]jPCT-AE. Welcome to jPCT-AE - a free 3D engine for android [online] Available: http://www.jpct.net/jpct-ae/.

[39] Margolis, RB, Tait, RC, Krause, SJ (1986) A Rating System for Use with Patient Pain Drawings. Pain 24: 57-65.

[40] Ohnmeiss, DD, Vanharanta, H, Ekholm, J (1999) Relationship of pain drawings to invasive tests assessing intervertebral disc pathology. Europ Spine J 8: 126-131.

[41]Pirhonen, A, Brewster, SA, Holguin, C. Gestural and Audio Metaphors as a Means of Control for Mobile Devices. In Proc ACM CHI, Minneapolis, MN, 2002, ACM Press, 291-298.

[42]Lumsden, J, Brewster, S. A Paradigm Shift: Alternative Interaction Techniques for Use with Mobile \& Wearable Devices. In Proc CASCON '03, Toronto, Ontario, Canada, 2003, IBM Press, 197-210.

[43] Fitzmaurice, GW, Zhai, S, Chignell, MH. (1993) Virtual Reality for Palmtop Computers. ACM Trans Info Systems 11 (8): 197-218.

[44]Vuzix, Corp. Wrap 920 Video Eyewear [online] Available: http://www.vuzix.com/consumer/products_wrap920.html\#overview

[45]Brooke, J (1996) SUS: a 'quick and dirty' usability scale. In: Jordan, PW, Thomas, B, Weerdmeester, BA, McClelland, AL Usability Evaluation in Industry. Taylor and Francis: London. 